\title{
Chitosan Pad, Cellulose Membrane, or Gelatin Sponge for Peridural Bleeding: An Efficacy Study on a Lumbar Laminectomized Rat Model
}

\author{
Surachai Sae-Jung, Punyawat Apiwatanakul \\ Department of Orthopaedics, Faculty of Medicine, Khon Kaen University, Khon Kaen, Thailand
}

\begin{abstract}
Study Design: Experimental study in an animal model.
Purpose: This study aims to evaluate the hemostatic properties of four common hemostatic materials including the chitosan clot pad, absorbable gelatin sponge, cellulose membrane, and gauze on peridural bleeding using a rat model.

Overview of Literature: Intraoperative bleeding during spinal surgery can lead to morbidities. Hemostatic materials have been developed, but the efficacy of these materials on peridural bleeding remains unclear.

Methods: Forty 8-week-old Sprague-Dawley rats were used in this study. Under adequate anesthesia, each rat was posteriorly dissected to their $\mathrm{L} 5$ and $\mathrm{L} 6$ spinous processes. Bleeding from muscles and soft tissue dissections was stopped before lumbar bone cutting. Immediately after the $L 5-L 6$ laminae were cut and removed, the rats were randomly allocated to receive one of the abovementioned hemostatic materials. All hemostatic materials were placed over the raw surface of cut bone and dura and changed every 60 seconds. The procedure was stopped when there was no further bleeding. Time to staunching and amount of bleeding were recorded for statistical analysis.

Results: The respective mean \pm standard deviation hemorrhage volume and time for the cellulose membrane, gelatin sponge, chitosan pad, and gauze were $1.19 \pm 0.44,1.03 \pm 0.72,0.96 \pm 0.57$, and $1.98 \pm 0.62 \mathrm{~mL}$, respectively, and $2.9 \pm 0.6,2.1 \pm 0.6,1.7 \pm 0.5$, and $2.9 \pm 1.0$ minutes, respectively. The overall bleeding volumes for the cellulose membrane, gelatin sponge, and chitosan pad were significantly lower than the overall bleeding volume for gauze.

Conclusions: Chitosan clot pads, gelatin sponges, and cellulose membranes have better hemostatic properties than gauze. The chitosan pad had the lowest average bleeding volume, followed by gelatin sponge and cellulose membrane.
\end{abstract}

Keywords: Hemostatics; Hemorrhage; Chitosan; Cellulose; Gelatin

\section{Introduction}

Bleeding control during spinal surgery is important because intraoperative bleeding and postoperative hematoma increase the risk of morbidity. Hemostatic modali- ties have been developed, including electrocautery and hemostatic materials (e.g., oxidized regenerated cellulose membrane, absorbable gelatin sponge, and chitosan clot pad). Although electrocautery and compressive dressings are effective in controlling large and visible bleeding

Received May 24, 2017; Accepted Aug 4, 2017

Corresponding author: Surachai Sae-Jung

Department of Orthopaedics, Faculty of Medicine, Khon Kaen University, Khon Kaen, 40002, Thailand

Tel: +66-43348398, Fax: +66-43348398, E-mail: sursea@kku.ac.th 
vessels, they also carry a high risk of complete occlusion of the vascular lumen [1]. They are also less effective for controlling diffuse capillary bleeding, which is common in spinal procedures. Absorbable gelatin sponges are used in most spinal surgeries for controlling capillary and venous bleeding [2]. The surgical cellulose pad-a membrane made of oxidized regenerated cellulose polymeris extensively used in oral and maxillofacial surgery to control intrabony arterial bleeds from the intraalveolar artery. A cellulose membrane is used around the dura to staunch bleeding and prevent peridural fibrosis following laminectomy. Chitosan-a natural polymer with a good safety profile-has been shown to control bleeding, improve wound healing, and reduce adhesion formation in animal and human studies. The use of these materials for spinal surgery is, however, unclear owing to insufficient data on their effectiveness and safety vis-à-vis bone bleeding near neural structures. Peridural bleeding is a major challenge for surgeons. The objective of this study was to compare the efficacy and safety of four commonly used hemostatic materials (cellulose membrane, gelatin sponge, chitosan clot pad, and gauze) in a lumbar laminectomized rat model.

\section{Materials and Methods}

\section{Study design and study population}

This study was reviewed and approved by the Animal Ethics Committee, Northeast Laboratory Animal Center, Khon Kaen University, based on the Ethic of Animal Experimentation of National Research Council of Thailand (AEKKU-NELAC 12/2557). All animal care, housing, and procedures were conducted at the institute's animal laboratory, which adheres to level 1 Animal Biosafety standards.

Forty 8 -week-old Sprague-Dawley rats, weighing 200$400 \mathrm{~g}$, were intraperitoneally anesthetized by injecting 60 $\mathrm{mg} / \mathrm{kg}$ pentobarbital in a $1-\mathrm{mL}$ syringe using a 24 -gauge needle; sterile technique was used. Once the rat's toe pinch response was lost, anesthetic depth was considered sufficient for surgery. Next, the rat was intraperitoneally injected with a single dose of cefazolin $150 \mathrm{mg}$ as a prophylactic antibiotic. The back skin, scrubbed with hibitane solution, was then shaved, taking care not to cut the skin. The rat was placed prone on an operative holder, its back painted with betadine solution, and a surgical drape ap- plied. The back skin was incised and retracted bilaterally. The paravertebral muscle was dissected and retracted, using a self-retaining retractor, to expose the L5-L6 spinous processes and laminae. The bleeding points were staunched and the L5-L6 laminae clearly identified. The L5-L6 spinous processes were cut and removed using a bone rongeur. Then the L5-L6 laminae were carefully cut and removed using a $1.0 \mathrm{~mm}$ Kerrison rongeur. Each viable rat without cerebrospinal fluid leakage was included in this study.

The computer-generated randomization code, kept in an opaque sealed envelope, was opened. The rat randomly received one of the four hemostatic materials (cut to $1 \mathrm{~cm}^{2}$ and sterilized): (1) cellulose membrane (Surgicel Original Absorbable Hemostat; Ethicon, Somerville, NJ, USA); (2) absorbable gelatin sponge (Spongostan; Ferrosan Medical Device, Soeborg, Denmark); (3) chitosan pad (AnsCare ChitoClot Pad; BenQ Materials Corp., Taoyuan, Taiwan); or (4) gauze. The retained hemorrhage from the laminectomy procedure was completely removed using the assigned hemostatic material. Thereafter, each assigned hemostatic material was placed over the dura and bony raw surface to stop the peridural bleeding. Every 60 seconds, the material was replaced with a fresh one until the active bleeding stopped.

Bleeding time and volume were recorded using a twodecimal digital clock and a two-decimal digital analytical balance, respectively. After the laminectomy area had no further bleeding, the hemostatic materials were removed and the surgical wounds were closed layer by layer. All subjects were given $300 \mathrm{mg} / \mathrm{kg}$ of Tylenol syrup orally three times a day for 2 days. The skin sutures were removed 7 days postoperatively. Adverse events observed by the investigator were also assessed and recorded as secondary outcomes.

\section{Statistical methods}

A power calculation indicated that 10 rats were needed in each group to have a $95 \%$ chance of detecting a $10 \%$ difference in mean bleeding volume. An analysis of variance (ANOVA) was used to analyze continuous variables, and the independent $t$-test with Bonferroni correction was used to analyze differences between each pair of interventions. The level of statistical significance was set at 0.05 . The data were analyzed using IBM SPSS Statistics ver. 20.0 (IBM Corp., Armonk, NY, USA), and post hoc power 
analysis of the study was calculated using $G^{\star}$ Power ver. 3.0.10 (Heinrich-Heine-Universität Düsseldorf, Düsseldorf, Germany; http://www.gpower.hhu.de/) [3].

\section{Results}

The average body weights of the males and females were $354.0 \pm 16.3$ and $214.9 \pm 8.2 \mathrm{~g}$, respectively; males were sig- nificantly heavier than females $(t[38]=33.98, p=0.001)$. The average bleeding volumes were $1.48 \pm 0.68 \mathrm{~mL}$ (males) and $1.11 \pm 0.70 \mathrm{~mL}$ (females) (no significant difference: $t$ $[38]=1.70, p=0.10$ ) (Table 1). The respective average bleeding durations were $2.7 \pm 1.0$ minutes (males) and $2.2 \pm 0.6$ minutes (females) (no significant difference: $t[38]=1.95$, $p=0.06$ ) (Table 1). The two-way ANOVA was conducted to analyzed the interaction between treatment group and

Table 1. Comparisons of the overall bleeding volume and bleeding time between male and female rats

\begin{tabular}{lcccc} 
Variable & Male $(\mathrm{n}=20)$ & Female $(\mathrm{n}=20)$ & Mean difference (95\% confidence interval) & $p$-value \\
Body weight $(\mathrm{g})$ & $354.0 \pm 16.3$ & $214.9 \pm 8.2$ & $139.1(130.8$ to 147.3$)$ & 0.001 \\
Bleeding volume $(\mathrm{mL})$ & $1.48 \pm 0.68$ & $1.11 \pm 0.70$ & $0.37(-0.07$ to 0.81$)$ & 0.10 \\
Bleeding time $(\mathrm{min})$ & $2.7 \pm 1.0$ & $2.2 \pm 0.6$ & $0.5(-0.02$ to 1.02$)$ & 0.06 \\
\hline
\end{tabular}

Values are presented as mean \pm standard deviation.

Table 2. The data of body weight, bleeding volume, and bleeding time according to the treatment groups

\begin{tabular}{|lcccc}
\hline Variable & Cellulose & Gelatin & Chitosan & Gauze \\
\hline Male (no.) & 5 & 5 & 5 & 5 \\
\hline Body weight $(\mathrm{g})$ & $361.6 \pm 17.2$ & $357.9 \pm 13.9$ & $350.3 \pm 18.1$ & $346.2 \pm 16.3$ \\
\hline Bleeding volume $(\mathrm{mL})$ & $1.40 \pm 0.44$ & $1.32 \pm 0.88$ & $1.24 \pm 0.70$ & $1.95 \pm 0.59$ \\
\hline Bleeding time $(\mathrm{min})$ & $3.2 \pm 0.4$ & $2.2 \pm 0.8$ & $1.8 \pm 0.4$ & $3.4 \pm 1.1$ \\
\hline Female (no.) & 5 & 5 & 5 & 5 \\
\hline Body weight $(\mathrm{g})$ & $209.6 \pm 4.4$ & $224.7 \pm 9.3$ & $214.4 \pm 7.3$ & $211.0 \pm 3.7$ \\
\hline Bleeding volume $(\mathrm{mL})$ & $0.99 \pm 0.37$ & $0.74 \pm 0.41$ & $0.68 \pm 0.20$ & $2.02 \pm 0.73$ \\
\hline Bleeding time $(\mathrm{min})$ & $2.6 \pm 0.5$ & $2.0 \pm 0.2$ & $1.6 \pm 0.5$ & $2.4 \pm 0.5$ \\
\hline Overall (no.) & 10 & 10 & 10 & 10 \\
\hline Body weight $(\mathrm{g})$ & $285.6 \pm 81.0$ & $291.3 \pm 71.1$ & $282.4 \pm 72.8$ & $278.6 \pm 72.1$ \\
\hline Bleeding volume $(\mathrm{mL})$ & $1.19 \pm 0.44$ & $1.03 \pm 0.72$ & $0.96 \pm 0.57$ & $1.98 \pm 0.62$ \\
\hline Bleeding time $(\mathrm{min})$ & $2.9 \pm 0.6$ & $2.1 \pm 0.6$ & $1.7 \pm 0.5$ & $2.9 \pm 1.0$ \\
\hline
\end{tabular}

Values are presented as number or mean \pm standard deviation.

Table 3. Post hoc comparisons of bleeding volume and bleeding time among the treatment groups

\begin{tabular}{|c|c|c|c|c|}
\hline \multirow{2}{*}{ Material 1 vs. 2} & \multicolumn{2}{|c|}{ Bleeding volume (mL) } & \multicolumn{2}{|c|}{ Bleeding time (min) } \\
\hline & $\operatorname{MD}(95 \% \mathrm{CI})$ & $p$-value & $\operatorname{MD}(95 \% \mathrm{Cl})$ & $p$-value \\
\hline Cellulose vs. control & $-0.79(-1.53$ to -0.05$)$ & 0.004 & 0.01 (-0.85 to 0.85$)$ & 1.00 \\
\hline Gelatin vs. control & $-0.95(-1.70$ to -0.21$)$ & 0.005 & $-0.8(-1.65$ to 0.05$)$ & 0.08 \\
\hline Chitosan vs. control & $-1.02(-1.77$ to -0.28$)$ & 0.001 & $-1.2(-2.05$ to -0.35$)$ & 0.002 \\
\hline Chitosan vs. cellulose & $-0.23(-0.98$ to 0.51$)$ & 0.32 & $-1.2(-2.05$ to -0.35$)$ & 0.002 \\
\hline Chitosan vs. gelatin & -0.07 (-0.81 to 0.68$)$ & 0.82 & $-0.4(-1.25$ to 0.45$)$ & 1.00 \\
\hline Cellulose vs. gelatin & 0.16 (-0.58 to 0.91$)$ & 0.55 & $0.8(-0.05$ to 1.65$)$ & 0.08 \\
\hline
\end{tabular}

Minus symbol (-) indicates the material 1 has less bleeding volume or shorter bleeding time than material 2. Control represents the gauze treatment group.

$\mathrm{MD}$, mean difference; $\mathrm{Cl}$, confidence interval. 


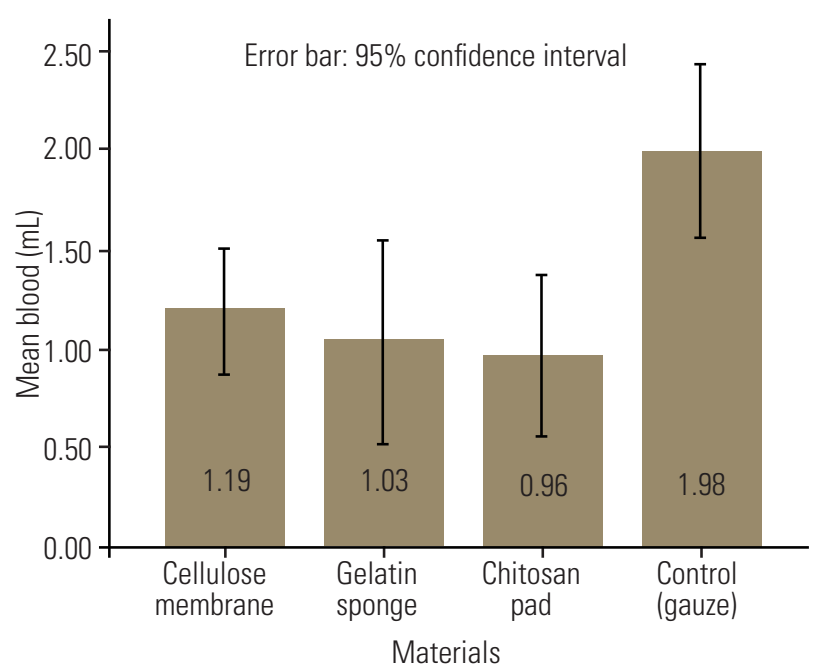

Fig. 1. The bar graph demonstrates mean of overall bleeding volume among cellulose membrane, gelatin sponge, chitosan pad, and gauze groups. Number within box represents mean bleeding volume $(\mathrm{mL})$

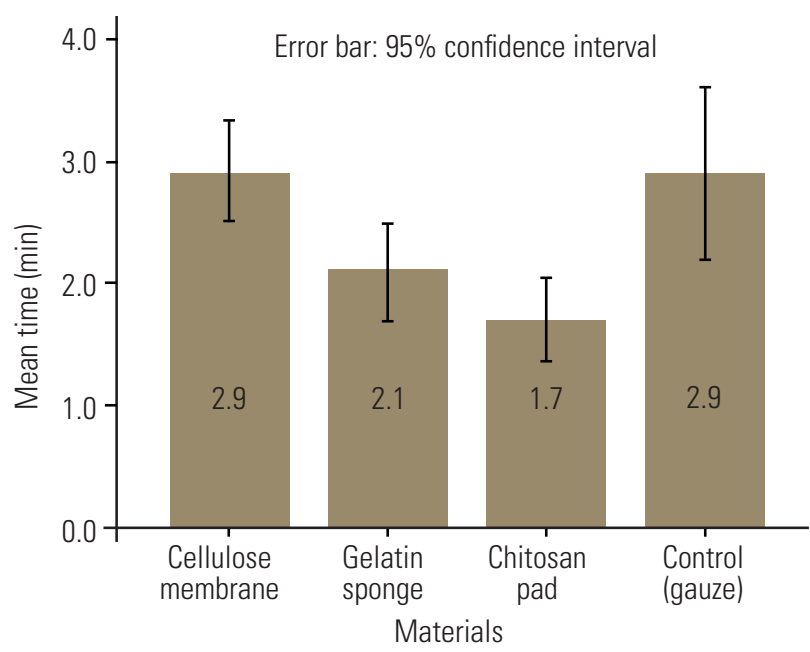

Fig. 2. The bar graph compares bleeding times among cellulose membrane, gelatin sponge, chitosan pad, and gauze. Number within box represents mean bleeding time (min).

sex on bleeding duration and bleeding volume and found that there were no significant difference among the treatment groups: $F[3,32]=0.89, p=0.46$ and $F[3,32]=0.67$, $p=0.58$, respectively (Table 2 ).

The average bleeding volumes after use of each of the four hemostatic materials were compared and significant differences were found $(F[3,36]=6.26, p=0.002)$. Post hoc comparisons between pairs of treatments, using independent $t$-test with Bonferroni correction, showed that the groups treated with cellulose membrane, absorbable gelatin sponge, and chitosan pad had lower bleeding volumes than the control group $(t[18]=3.28, p=0.004 ; t[18]=3.17$, $p=0.005$; and $t(18)=3.83, p=0.001$, respectively) (Table 3 , Fig. 1).

According to ANOVA, there were significant differences in average bleeding time among the treatment groups ( $F$ $[3,36]=7.71, p=0.0001)$. Between the pairs of treatment, chitosan had significantly less bleeding time than the control ( $t[18]=3.43, p=0.002)$ (Table 3, Fig. 2). However, there were no statistically significant differences among the cellulose, gelatin, and control groups (Table 3, Fig. 2). Post hoc power analyses of the study were 0.94 for bleeding volume and 0.97 for bleeding time.

\section{Discussion}

The hemostatic materials cellulose membrane, absorbable gelatin sponge, and chitosan pad staunch peridural bleeding better than gauze (or the conventional method). Cellulose membrane and gelatin sponge are absorbents. Oxidized regenerated cellulose membrane is a homopolysaccharide, comprising glucopyranose polymerized through $\beta$-glucosidic bonds [4]. Its acidity arises from a carboxyl group, which provides a primary local bactericidal hemostyptic action. Secondary platelet activation results in a platelet plug [5] via physical scaffolding [6].

The absorbable gelatin sponge is biodegradable, offwhite, and porous. It is prepared from dried and sterilized porcine skin gelatin. Jenkins et al. [7] suggest that the hemostatic properties of this sponge result from damage resulting to platelets that enter and contact the sponge's interstices. Thromboplastin is thus released from these platelets initiating a clotting cascade. Thus, the gelatin sponge has no direct effect on clotting. The sponge can absorb as much as 40 times its weight in blood or fluid and can expand to approximately $200 \%$ [6].

The chitosan clot pad-a polysaccharide polymer of glucosamine-is a hemostatic electropositive spongelike material that allows binding of normally negatively charged red blood cells and platelets, facilitating blood clot formation. In addition, the electropositive surface attracts other chemotactic factors involved in clotting.

We compared cellulose membrane, gelatin sponge, chitosan clot pad, and gauze as the control material. All were cut to the same size and placed over the laminar raw surface and dura without applying pressure to avoid a pressure effect, which would cause dural sac compression and confound the hemorrhage volume and/or time. Regarding physical properties, absorption and adsorption are the 
two main hemostatic properties. Absorption reflects the ability of a material to "soak up" blood or other fluids, while adsorption is the adherence of fluid or blood to the material surface. We used bleeding time and volume to test the in vivo efficacy of the hemostatic materials because these two outcome measurements reflect the clinical implications of material use, i.e., the duration and volume of bleeding during spinal surgery. Schreiber and Neveleff [8] also recommended bleeding time, severity of bleeding, and possible adverse events as critical factors in hemostat selection. We included both female and male rats in our study because the Food and Drug Administration now requires the inclusion of both females and males in clinical trials.

Due to the absorption and adsorption effects of the materials, the chitosan clot pad had significantly less hemorrhage volume than the control (gauze), possibly because the cationic surface of chitosan enhances platelet aggregation. Notwithstanding, the bleeding volume was not significantly different for cellulose membrane or gelatin sponge, possibly because of the acceleration of platelet aggregation by the cellulose membrane and gelatin sponge from their structural lattice. Paradoxically, they possess potent absorption properties, soaking up greater overall hemorrhage volumes, although these volumes were not statistically different from those absorbed by gauze. We found the hemorrhage volume within the materials depended on their absorption property, whereas bleeding time was more a function of material surface adsorption.

In the current study, bleeding time was the time between bone wounding and bleeding cessation. After the bony injury, the platelets adhere, aggregate, and form a platelet plug. Bleeding time measures the ability of platelets to staunch bleeding; consequently, bleeding time reflects the platelet function and platelet number. As such, the chitosan clot pad had significantly shorter bleeding time than the control. These might be from the platelet aggregation and platelet plug that occurred when the negatively charged platelet surface contacted the positively charged surface of the chitosan fibers (Fig. 3). For cellulose membrane and gelatin sponge, the bleeding time trended shorter than the control, although this difference did not rise to the level of statistical significance. This may be because cellulose and gelatin resemble platelet
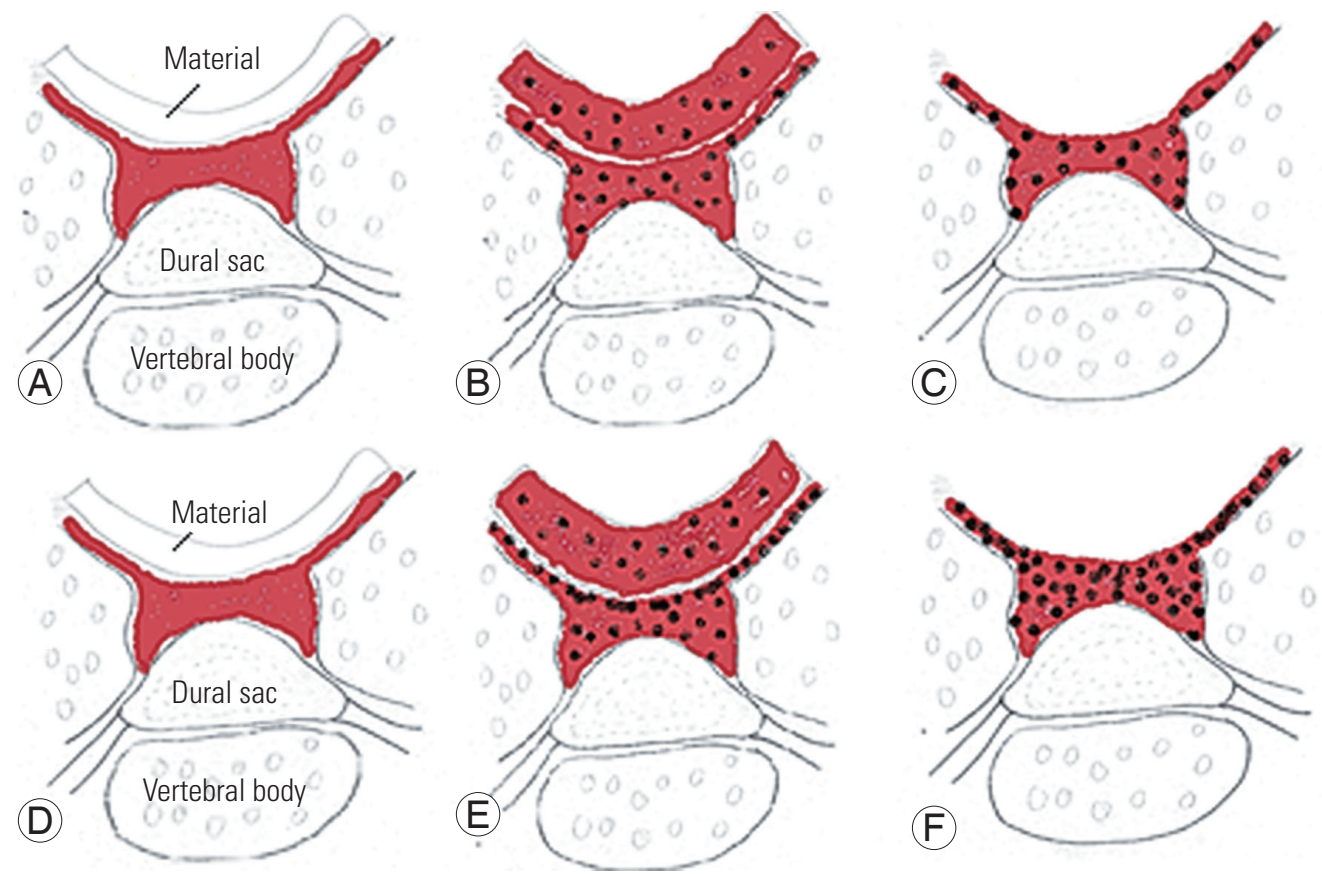

Fig. 3. The drawing demonstrates platelet aggregation of $(\mathbf{A}-\mathbf{C})$ absorbable gelatin sponge or cellulose membrane, and (D-F) nonabsorbable chitosan clot pad. (A) The absorbable material (gelatin sponge or cellulose membrane) is placed over the bleeding of bone and dural sac. (B) The blood and platelet are absorbed into the material, the material is expanded. (C) After material removal: the remnant of the scared platelet plug with some hemorrhage, which require the new piece of hemostatic material. (D) The chitosan clot pad is placed over the bleeding bone and dural sac. (E) The blood and platelet are absorbed into the material, note (the platelets are highly adsorbed at the chitosan surface. (F) After material removal: the remnant of condensed platelet plug with minimal bone hemorrhage. Red shading and black dots represent blood and platelets, respectively. 
aggregation, based on their physical properties, which may also enable blood absorption. Therefore, when the blood soaked cellulose membrane or gelatin sponge were removed, the platelet aggregation within these materials and the platelet plug on the raw bone surface and dural sac are removed and new bleeding occurred (Fig. 3). This material was different than the chitosan clot pad, which absorbs some blood, but only a thin layer of platelet aggregation. The thickness of the residual platelet layer can plug the epidural and bony bleeding point more than any other mechanism.

Bleeding time associated with the chitosan clot pad was shorter than the other tested materials. As for hemorrhage volume, there were no differences among the three testing materials. We found that most of the hemorrhage was absorbed within these materials (Fig. 3). Hemorrhage time appears to depend on the rate of platelet aggregation on the raw surface of the bleeding bone and dura, while hemorrhage volume depends on blood absorption within the material. The cellulose membrane and gelatin sponge primarily possess an absorption effect, so the blood and platelets are highly distributed within the material. After removal, the hemorrhage continues and the new piece of the hemostatic material is required. The chitosan possesses both adsorption and absorption effects. Blood fluid is absorbed into the chitosan pad; however, the platelets are adsorbed as a condensed platelet aggregation at the surface of the material-bone interface, resulting in less hemorrhage volume after the chitosan pad was removed (Fig. 3). For the overall hemorrhage volume, the chitosan clot pad was not significantly different, compared to the cellulose membrane or gelatin sponge. This may be because the absorption property of the chitosan pad, which can absorb and expand 30 times, or more, of its dried volume. Based on bleeding time and bleeding volume, we compared and summarized the adsorption and absorption properties of the test materials as shown in Table 4.

There were no immediate adverse events correlated with the use of any testing material. However, the side effect of the retaining material within epidural space cannot be measured due to the material removal after cessation of bleeding. This is the limitation of the current study.

Bone wax is the other hemostatic material used for bone bleeding. However, bone wax is not suitable for peridural space bleeding where blood originates from both the cut surface of the bone and epidural vessels [9]; a previous study demonstrated that the bone wax exerted direct pressure on neural tissue [10]. Nooh et al. [11] recommended complete removal of bone wax because it induced inflammation and delayed bone healing. Moreover, for the small $\left(1 \mathrm{~cm}^{2}\right)$ laminectomized area of rat, while prone, there was elevated risk of neurological compression when we use the bone wax as hemostatic material. In many scenarios (e.g., minimal invasive spinal decompression), bone wax is difficult to use due to risk of neurological compression. So, we did not include this material in the study.

In conclusion, all testing materials had more success staunching bleeding than gauze, and all materials could be used to staunch bleeding bone after spinal surgery, albeit with different mechanisms of hemostasis. The nonabsorbable chitosan clot pads must be removed while the absorbable materials, cellulose membrane, and gelatin sponge, can be retained in the operative field. The expandable size of the absorbable material can, however, cause pressure affecting nearby structures. Previous studies reported that the oxidized regenerated cellulose membrane contributed to granuloma after brain surgery [12-14], migrated into the spinal canal after esophagectomy [15], caused paraplegia after thoracotomy [16] and caused cauda equina syndrome after lumbar microdiscectomy [17]. For the absorbable gelatin sponge, earlier studies reported blood saturated gelatin sponges formed a gelatinous mass compressing the brain [18], spinal cord [19], nerve root [20] or cauda equina $[17,21]$; following spinal surgery. Consequently, these should be used with care if near a neural structure. We recommend that cellulose membrane and absorbable gelatin sponge are removed when bleeding stops.

Table 4. Summary of adsorption and absorption properties of the tested hemostatic materials

\begin{tabular}{lcccc} 
Property & Cellulose membrane & Gelatin sponge & Chitosan pad & Gauze \\
Absorption & +++ & +++ & +++ & +++ \\
Adsorption & $0 /+$ & $0 /++$ & +++ & $0 /+$ \\
\hline
\end{tabular}

Zero symbol (0) represents neutral effect. The plus symbol (+) represents the positive effect. 


\section{Conclusions}

The chitosan clot pad, cellulose membrane, and gelatin sponge had better hemostatic properties than the gauze when used to staunch peridural bleeding in a laminectomized rat model. Chitosan pad had the lowest average bleeding volume followed by gelatin sponge, cellulose membrane, and gauze, respectively. Each of the three can be clinically used as a peridural hemostatic material without serious adverse events.

\section{Conflict of Interest}

No potential conflict of interest relevant to this article was reported.

\section{Acknowledgments}

The study was supported by Research Scholarship, TRB Chemedica, Thailand. The authors thank Mr. Bryan Roderick Hamman for assistance with the English-language presentation of the manuscript through the aegis of the Publication Clinic Khon Kaen University, Thailand.

\section{References}

1. Sabel M, Stummer W. The use of local agents: Surgicel and Surgifoam. Eur Spine J 2004;13 Suppl 1:S97-101.

2. Gerald AG. Update on hemostasis: neurosurgery. Surgery 2007;142(4 Suppl):S55-60.

3. Faul F, Erdfelder E, Lang AG, Buchner A. G*Power 3: a flexible statistical power analysis program for the social, behavioral, and biomedical sciences. Behav Res Methods 2007;39:175-91.

4. Pierce AM, Wiebkin OW, Wilson DF. Surgicel: its fate following implantation. J Oral Pathol 1984;13:661-70.

5. Miller JM, Jackson DA, Collier CS. An investigation of the chemical reactions of oxidized regenerated cellulose. Exp Med Surg 1961;19:196-201.

6. Sundaram CP, Keenan AC. Evolution of hemostatic agents in surgical practice. Indian J Urol 2010;26:374-8.

7. Jenkins HP, Owen H, Senz E, Jampolis RW. Control of hemorrhage from wounds of the heart by the gelatin sponge "patch" technic: a new experimental method. Ann Surg 1947;126:973-89.

8. Schreiber MA, Neveleff DJ. Achieving hemostasis with topical hemostats: making clinically and economically appropriate decisions in the surgical and trauma settings. AORN J 2011;94:S1-20.

9. Yi S, Yoon DH, Kim KN, Kim SH, Shin HC. Postoperative spinal epidural hematoma: risk factor and clinical outcome. Yonsei Med J 2006;47:326-32.

10. Karabekır HS, Korkmaz S. Residue bone wax simulating spinal tumour: a case report. Turk Neurosurg 2010;20:524-6.

11. Nooh N, Abdullah WA, Grawish Mel-A, Ramalingam S, Javed F, Al-Hezaimi $\mathrm{K}$. The effects of surgicel and bone wax hemostatic agents on bone healing: an experimental study. Indian J Orthop 2014;48:319-25.

12. Sandhu GS, Elexpuru-Camiruaga JA, Buckley S. Oxidized cellulose (Surgicel) granulomata mimicking tumour recurrence. Br J Neurosurg 1996;10:617-9.

13. Ito $\mathrm{H}$, Onishi $\mathrm{H}$, Shoin $\mathrm{K}$, Nagatani H. Granuloma caused by oxidized cellulose following craniotomy. Acta Neurochir (Wien) 1989;100:70-3.

14. Lin B, Yang H, Cui M, Li Y, Yu J. Surgicel application in intracranial hemorrhage surgery contributed to giant-cell granuloma in a patient with hypertension: case report and review of the literature. World J Surg Oncol 2014;12:101.

15. Dua S, Purandare NC, Merchant NH, Pramesh CS. Oxidised regenerated cellulose: an unusual cause of paraplegia following oesophagectomy. Interact Cardiovasc Thorac Surg 2010;10:833-5.

16. Attar S, Hankins JR, Turney SZ, Krasna MJ, McLaughlin JS. Paraplegia after thoracotomy: report of five cases and review of the literature. Ann Thorac Surg 1995;59:1410-5.

17. Bessette MC, Mesfin A. Cauda equina syndrome caused by retained hemostatic agents. J Clin Neurosci 2015;22:1518-20.

18. Herndon JH, Grillo HC, Riseborough EJ, Rich JC Jr. Compression of the brain and spinal cord following use of gelfoam. Arch Surg 1972;104:107.

19. Alander DH, Stauffer ES. Gelfoam-induced acute quadriparesis after cervical decompression and fusion. Spine (Phila Pa 1976) 1995;20:970-1..

20. Kao FC, Tsai TT, Chen LH, et al. Symptomatic epidural hematoma after lumbar decompression surgery. Eur Spine J 2015;24:348-57.

21. Tan WC, Liao WJ, Liu JT. Spongostan-induced cauda equina syndrome after lumbar discectomy: report of a case. Formos J Surg 2008;41:99-103. 